\title{
Awareness and timing of pelvic floor muscle contraction, pelvic exercises and rehabilitation of pelvic floor in lifelong premature ejaculation: 5 years experience
}

\author{
Giuseppe La Pera \\ Department of Urology, San Camillo Forlanini Hospital, Rome, Italy.
}

\begin{abstract}
Summary Objectives: To assess the cure rate of patients with premature ejaculation who underwent a treatment involving: 1) awareness of the pelvic floor muscles 2) learning the timing of execution and maintenance of contraction of the pelvic floor muscles during the sensation of the pre-orgasmic phase 3) pelvic floor rehabilitation (bio feed back, pelvic exercises and electrostimulation).

Materials and methods: We recruited 78 patients with lifelong premature ejaculation who completed the training. The patients were informed of the role of the pelvic floor. They were taught to carry out the execution and maintenance of contraction of the pelvic floor muscles during the sensation of the pre-orgasmic phase to control the ejaculatory reflex. In order to improve the awareness, the tone and the endurance of the pelvic floor muscles, patients were treated with the rehabilitation of pelvic floor (RPF) consisting mainly in biofeedback, pelvic exercises and in some cases also in electro-stimulation (ES). The training was carried out for a period of about 2-6 months with an average of 2-5 visits per cycle.
\end{abstract}

Results: $54 \%$ of patients who completed the training were cured of premature ejaculation and learned over time to be able to postpone the ejaculation reflex. In a subgroup of 26 patients was also measured the IELT which on the average increased from $<2$ minutes to $>10$ minutes. The best results occurred mainly in patients aged less than 35 where the cure rate was $65 \%$. There were no side effects.

Conclusions: In this study, approximately half of patients with premature ejaculation were cured after applying the above treatment.This therapy, necessitates a fairly long period of time (2-6 months) and a great commitment on the part of the patient, nevertheless it can be a valid and effective treatment for patients with premature ejaculation. This treatment makes the patient independent in that he is not bound to specific times for taking medication. Furthermore there are no side effects and this therapy is particularly effective in young males.

KEY WORDS: Premature ejaculation; Awareness; Timing; Pelvic floor muscles; Pre-orgasmic sensation.

Submitted 26 May 2014; Accepted 16 June 2014

\section{INTRODUCTION}

The pelvic floor rehabilitation (PFR) consisting in biofeedback, pelvic exercises (kinesis-therapy) and pelvic floor electrical stimulation in the treatment of lifelong premature ejaculation has been introduced since 1996 (1) and was later confirmed by other authors (2).

Since then, from 2008, compared to the previously described technique, I introduced some changes in the therapeutic protocol:

1. adopting the new definition of premature ejaculation (PE) of the ESSM (3);

2. introducing into the protocol the awareness of the pelvic floor muscles (4);

3. teaching patients when to execute and maintain the contraction of the pelvic floor muscles. In order to inhibit the ejaculatory reflex this contraction must occur during the sensation of the pre-orgasmic phase.

As regards the changes to the protocol of training, these are based on the observation that patients with premature ejaculation very often are not aware of the role of the pelvic floor muscles (4). Therefore some patients may suffer premature ejaculation simply because they do not know what to do or are not able to make a selective effective contraction of the pelvic floor and not because of an early arrival of the stimulus. The purpose of this study was to evaluate the cure rates in an unselected population of patients with lifelong premature ejaculation undergoing rehabilitation treatment of the pelvic floor who have been taught the role of the pelvic floor and the timing of contraction of these muscles.

\section{Materials AND METHOdS}

Recruitment of patients

We retrospectively reviewed the charts of 108 patients with lifelong premature ejaculation. Out of them 78 patients (72\%) completed the protocol training. Patients with erectile dysfunction and patients with signs of prostatitis were excluded. The majority of patients excluded from treatment or who did not complete the protocol preferred an immediate drug treatment instead of a treatment that

No conflict of interest declared. 
required numerous sessions spread over a period of several months. The average age was $41+/-8$ and a median age of 40 (range 18 to 64).

\section{Definition of a patient with premature ejaculation}

The definition of a patient with premature ejaculation refers to the definition adopted by the ESSM 2008 (3); all patients had the IELT (5) in less than one minute and a PEDT (6) test $>11$. In the hormone screening was found only one patient with hyperthyroidism; in all the other patients, the hormonal values of the thyroid function were always found normal. Eight patients had a significantly low testosterone. In all these cases of hormonal alteration, the hormonal treatment implemented to correct hypogonadism or hyperthyroidism did not change the symptoms of premature ejaculation.

\section{Description of the technique}

The technique to inhibit the ejaculatory reflex and to teach the patient how to take greater control consists of four steps. The 4 steps are:

1) Have the patient become aware of the existence of the pelvic floor muscles;

2) Teach the patient the selective contraction of the muscles of the pelvic floor;

3) Teach the patient and have him try out the timing of the execution and maintenance of contraction of the muscles of the pelvic floor during the sensation of the pre-orgasmic phase;

4) Reinforcement of the pelvic floor muscles.

\section{Awareness of the role of pelvic floor muscles}

At the beginning of treatment, all patients were made aware of the role of the pelvic floor through a test described in 2012 (4). In practice, the patient lying down is asked to contract his pelvic floor muscles while performing a digital rectal examination. At the end of this procedure the patient is asked whether he is aware that the contraction of the muscles of the pelvic floor is able to inhibit the ejaculatory reflex. A more accurate description of this technique and the demonstration of its validation was previously published (4)

\section{Pelvic floor rehabilitation (PFR)}

To learn how to selectively use the muscles of the pelvic floor, i.e. without activating antagonist muscles such as the abdominal muscles, we used the technique of PFR that involves biofeedback, pelvic exercises and in some cases electro-stimulation. This technique has already been described for fecal and urinary incontinence and in the treatment of premature ejaculation (1). The PFR not only reinforces the tone and strength of contraction of the pelvic floor muscles but at the same time allows the patient to better understand the role of these muscles and acquire the capacity to perform the contraction selectively. A more detailed description of the technique was previously published in 1996 (1).

\section{Nearing the orgasmic phase and timing}

\section{of the contraction of the pelvic floor muscles}

One of the techniques used up to now for the treatment of premature ejaculation has historically been that of "stop and start" proposed by Masters and Johnson since the 1960's (7). Basically it consists in stopping the stimulation when the excitation reaches the pre-orgasmic phase and and when the ejaculation is about to arrive. The innovation we have brought to our technique is that of adding, during the sensation of the pre-orgasmic phase, the execution and maintenance of contraction of the muscles of the pelvic floor until the sensation of upcoming orgasm has passed.

To obtain this result obviously the first step is to be informed of the existence and the role of the pelvic floor muscles. The second moment is to be able to carry out the contraction of the muscles of the pelvic floor in a selective manner and this can be obtained through biofeedback. The third element consists of the selective and coordinated contraction of the pelvic floor muscles to be carried out at the very pre-orgasmic moment before ejaculation becomes irreversible. This technique starts from the assumption that the contraction, performed in a coordinated and timely way, would be able to inhibit the ejaculatory reflex similarly to what happens in the bladder where the contraction of the pelvic floor muscles if done at a certain moment is capable of delaying the urge of urination through inhibition of detrusor contraction (8).

In order to teach the patient the "timing" of the contraction and have him figure out at what moment of preorgasmic sensation it is still possible to inhibit the ejaculatory reflex, exercises of masturbation are assigned to do at home. In these focusing exercises the patient begins to masturbate and gets to a stage of pre-orgasmic excitement; through "trial and error" he must learn to be able to recognize in what moment during the sensation of pre-orgasmic excitement it is still possible to inhibit the ejaculatory reflex in stopping masturbation, contracting the muscles of the pelvic floor and maintaining the contraction until the sensation of imminent orgasm ceases. Once the patient has achieved inhibition of the ejaculatory reflex and the sensation of imminent orgasm ceases the patient resumes masturbation and in the same session repeats this cycle 3 or 4 times. After 4 or 5 cycles the patient can let himself reach ejaculation. The results of this training and in particular the way the contraction is performed and its effectiveness in postponing the ejaculatory reflex are discussed with the patient once a month; any doubts are cleared up and further improvements made.

\section{Measurement of results and definition of healing}

Patients were considered cured if able to control their ejaculatory reflex and when they pass from a PDET score above 10 to one equal or less than 8 . Furthermore in one group of patients was evaluated the IELT (5) both before and at the end the training.

\section{RESULtS}

All patients at the end of the training due to awareness tests (4) have become conscious of the role of the pelvic floor in the control of ejaculation; 43 patients or $55 \%$ of all those who completed the training were cured of premature ejaculation and learned to be able to postpone the moment of ejaculation. In a subgroup of 26 patients was also measured the IELT which on the average went 
from $<2$ minutes to $>10$ minutes. The best results occurred mainly in patients aged less than 35 where the cure rate was $65 \%$. In the course of more than five years, there were no side effects in the whole sample involved. Thirty-five patients (45\%) after a minimum period of three months did not demonstrate any benefit from the technique proposed here and drug therapy was begun with them. The non-response to treatment was mainly due to the fact that the patient was not able to selectively contract the pelvic floor muscles or could not recognize the moment to make the contraction. Among these 35 patients, however, there were 12 in which the patient, even though making a regular and effective contraction of the muscles of the pelvic floor and even recognizing the moment when it was necessary to perform the contraction, had no beneficial effect on the length of ejaculatory latency. Probably this subset of patients is that in which premature ejaculation is the consequence of the early arrival of the ejaculatory stimulus.

\section{Discussion}

Awareness of the role of the pelvic floor muscles, the PFR and learning to recognize the timing of the execution and maintenance of contraction of the pelvic floor muscles are the crucial elements of this technique in order to inhibit and improve control over the ejaculatory reflex. The limit of this method is that it takes a few months for the patient to understand the dynamics of the sequence of events, learn control over the ejaculatory reflex and naturally carry it during sexual intercourse. In many family and clinical situations there is not always a long time available and thus the patient may request something faster such as drug therapy available today. Another limitation of this technique is that not all patients are able to perform a selective contraction of the pelvic floor muscles without activating other antagonist muscles or are not able to recognize the sensation that precedes the inevitability of ejaculatory reflex in order to perform the contraction of the muscles of the pelvic floor to block and inhibit ejaculation.

In the course of this experience, we observed that although some patients were able to correctly perform the contraction of the muscles of the pelvic floor this technique did not prove effective. This percentage of patients is typically around $15 \%$. These limits are, however, largely offset by the fact that recovery from premature ejaculation with this technique brings the patient around to be "independent", not influencing his sexual activity by taking a drug and not exposing him to potential side effects or drug interactions. These results confirm moreover the fact that the population of patients with premature ejaculation is a heterogeneous population with different etiological factors. There are not only patients in whom there is an early arrival of the ejaculatory reflex.

The positive results of this technique, which does not act on the arrival of the ejaculatory reflex but only on the control, suggest the hypothesis that premature ejaculation, in some cases, may be due to another four causes:

- lack of knowledge of the fact that is necessary to contract the pelvic floor muscles;

- inability to contract these muscles;
- inability to know how to recognize at what moment during the sensation of the pre-orgasmic phase it is still possible to block the ejaculatory reflex;

- result of a disease of the muscles of the pelvic floor.

These data raise therefore the urgent need not only to reassess the definition of premature ejaculation or at least redefine the concepts that define it but to re-evaluate the criteria for inclusion or exclusion in therapeutic trials on premature ejaculation taking into consideration the variables of awareness and neuromuscular coordination of the pelvic floor, the perception of the arrival of the ejaculatory reflex and diseases of the pelvic floor muscles.

\section{Conclusions}

In this study, approximately half of the patients with premature ejaculation were cured after they learned role and use of the muscles of the pelvic floor, having learned the timing of execution and maintenance of contraction of the pelvic floor muscles during the sensation of the pre-orgasmic phase and having undergone PFR. Despite the fact that this therapy in order to achieve positive results requires a fairly long period of time (2-6 months) and a greater commitment on the patient's part, it can be a valid and effective treatment to offer a patient with premature ejaculation. This treatment makes the patient independent in that he is not bound to specific times for taking medication. Furthermore there are no side effects and this therapy is particularly effective in young males.

\section{REFERENCES}

1. La Pera G, Nicastro A. A new treatment for premature ejaculation: the rehabilitation of the pelvic floor. J Sex Marital Ther. 1996; 22:22-6.

2. Pastore AL, Palleschi G, Leto A, et al. A prospective randomized study to compare pelvic floor rehabilitation and dapoxetine for treatment of lifelong premature ejaculation. Int J Androl. 2012; 35:528-33.

3. McMahon CG, Althof SE, Waldinger MD, et al. An evidence-based definition of lifelong premature ejaculation: report of the International Society for Sexual Medicine (ISSM) ad hoc committee for the definition of premature ejaculation. J Sex Med. 2008; 5:1590-606.

4. La Pera G. Awareness of the role of the pelvic floor muscles in controlling the ejaculatory reflex: preliminary results. Arch Ital Urol Androl. 2012; 84:74-8.

5. Waldinger MD, Hengeveld MW, Zwinderman AH, Olivier B. An empirical operationalization study of DSM-IV diagnostic criteria for premature ejaculation. Int J Psychiatry Clin Pract 1998; 2:287-293.

6. Symonds T, Perelman MA, Althof S, et al. Development and validation of a premature ejaculation diagnostic tool. Eur Urol. 2007; 52:565-73.

7. Masters WH, Johnson VE. Premature ejaculation. In: Human Sexual Inadequacy. Boston, Mass: Little Brown \& Company. 1970; 92-115.

8. Burgio KL, et al. Behavioral vs drug treatment for urge urinary incontinence in older women. JAMA 1998; 280:1995-2000.

\section{Correspondence}

Giuseppe La Pera, MD (Corresponding Author)

lapera@libero.it

Dept of Urology, San Camillo Forlanini Hospital, Rome, Italy 